\title{
Preventing Futile Liver Resection: A Risk-Based Approach to Surgical Selection in Major Hepatectomy for Colorectal Cancer
}

\author{
Marc W. Fromer, MD, Charles R. Scoggins, MD, MBA, Michael E. Egger, MD, MPH, Prejesh Philips, MD, \\ Kelly M. McMasters, MD, PhD, and Robert C. G. Martin II, MD, PhD \\ Division of Surgical Oncology, Department of Surgery, University of Louisville, Louisville, KY
}

\begin{abstract}
Background. Early recurrence following liver resection for metastatic colorectal cancer generally portends poor survival. We sought to identify factors associated with early disease recurrence after major hepatectomy for metastatic colorectal cancer in order to improve patient selection and prevent futile hepatectomy.

Methods. Sequential major (four or more segments) liver resections performed for metastatic colorectal cancer between 1995 and 2019 were selected from our prospectively maintained database. Univariate analyses, multivariable regression modelling, and survival analyses were used to identify predictors of futile resection (recurrence within 6 months of hepatectomy).

Results. Of 259 patients included, the median age was 61.3 years (interquartile range [IQR] 15.3) and the median number of liver tumors was 3.0 (IQR 2.0); $78.0 \%$ of patients received prehepatectomy chemotherapy. Surgeries were right $(56.4 \%)$, left $(19.3 \%)$, and extended hepatectomy $(24.3 \%)$. Futile resection occurred in $26(12.6 \%)$ patients. Margin positivity was similar in the futile resection group compared with the non-futile resection group (11.5\% vs. $11.4 \%)$. Extrahepatic disease that disappeared with chemotherapy was present in $23.1 \%$ of patients with a futile resection and $7.2 \%$ of those without $(p=0.019)$.
\end{abstract}

This work was presented at Digestive Disease Week 2020-SSAT; 2 May 2020; Chicago, IL, USA (virtual meeting due to COVID-19).

(C) Society of Surgical Oncology 2021

First Received: 7 June 2021

Accepted: 13 August 2021;

Published Online: 14 September 2021

R. C. G. Martin II, MD, PhD

e-mail: Robert.Martin@louisville.edu
After multivariable regression, the factors predictive of futile resection were extrahepatic disease (odds ratio [OR] 5.6; $p=0.004$ ), more than three liver lesions (OR 4.9; $p=0.001$ ), and extended hepatectomy (OR 2.6; $p=0.038$ ). Notably, $70.8 \%$ of futile recurrences occurred within the liver remnant and $20.8 \%$ were pulmonary metastases. Overall survival was 11.7 months $(95 \%$ confidence interval $[\mathrm{CI}]$ 7.1-16.2) for the futile resection cohort versus 45.6 (95\% CI 39.1-52.1) for non-futile hepatectomies $(p<0.001)$.

Conclusions. Futile hepatic resection can be predicted based on preoperative factors and carries a poor prognosis. Improved risk stratification for futility will aid in patient selection and treatment discussions.

Colorectal cancer is the third most common malignancy in the US and the second most lethal. ${ }^{1}$ Although validated screening measures and early endoscopic intervention have helped to decrease the incidence in Western nations, colorectal cancer remains a major public health concern. While local disease control can be attained in the vast majority of cases, metastatic dissemination of colorectal cancer is much more frequently responsible for loss of life. Up to $25 \%$ of all patients with the disease will ultimately develop metastases to the liver. ${ }^{2,3}$ Successful management of oligometastatic disease has allowed a subset of stage IV patients to survive for extended periods. Studies examining resection of isolated liver metastases have consistently demonstrated improved survival and potential long-term remission. $^{4-6}$

Determining which patients will benefit from hepatic metastasectomy in colorectal cancer remains challenging. Several reports have identified factors that predict recurrence or long-term survival after resection of liver metastases. ${ }^{7-12}$ Perhaps the best known is the risk model 
referred to as the 'Clinical Risk Score' by Fong et al. ${ }^{7}$ This score considers several clinical factors to predict risk of recurrence: preoperative serum carcinoembryonic antigen (CEA) concentration $>200 \mathrm{ng} / \mathrm{mL}$, a liver lesion $>5 \mathrm{~cm}$ in diameter, more than one liver lesion, a lymph node-positive colorectal primary tumor, and a colorectal-to-hepatic disease-free interval (DFI) of $<12$ months. This model was intended for risk stratification of recurrence after hepatectomy to guide the use of adjuvant therapy, but not necessarily for preoperative surgical selection.

In the modern era, simply predicting recurrence after hepatectomy may be less useful for surgical decision making, as meaningful prolongation of survival is still possible even with recurrences. Although disease eradication is possible with an initial hepatic resection, the vast majority (over $75 \%$ ) of patients will unfortunately experience recurrent disease. ${ }^{5}$ Indeed, long DFIs after liver resections may well justify these operations in spite of eventual recurrence events. In addition, multi-agent systemic therapy regimens have increased expected survival in stage IV disease and can be effective for post-hepatectomy recurrences. ${ }^{13-16}$ Local interventions, such as hepatic arterial therapies and direct ablative therapies of liver lesions, are also available as adjuncts for the treatment of liver-only or liver-dominant recurrences. ${ }^{17,18}$ While none of these interventions have been shown to be oncologically superior to resection, they can generally be administered with less morbidity and can preserve functional liver parenchyma. In unresectable cases, these therapies can, on occasion, sufficiently decrease tumor burden to allow for surgical resection. ${ }^{19}$ With these many options in play, many post-hepatectomy recurrences can be effectively managed with some combination of local or systemic therapies. Resections are just one element in a long-term multimodal treatment plan, making the pure utility of surgery increasingly difficult to study. ${ }^{20-22}$

If a major (four or more segments) liver resection is required to clear metastatic disease, there many potential consequences of this operation that must be considered. First, large parenchymal resections have the potential to create a liver remnant too compromised to tolerate additional local therapies. Moreover, multi-agent chemotherapy regimens are hepatotoxic and can result in a considerable degree of liver dysfunction within the metastatic population. $^{23}$ Major resections also carry a significant perioperative morbidity and mortality rate that must be factored into any surgical decision. ${ }^{24}$ Widespread micrometastatic disease present at the time of major liver resection may additionally result in rapid progression after operation. Major liver resection should be performed in patients where the risks are justified by a reasonable period of disease control.
Appropriate patient selection is critical to prevent a futile major hepatectomy in metastatic colorectal cancer. We defined an early disease recurrence-within 6 months of operation-as clear failure to achieve meaningful disease control. Presumably, major liver resection is carried out with curative intent or at least to render the patient disease-free for a period of time worthy of the considerable procedural downsides. The early recurrence group assumed all of the surgical risk without a discernable disease control benefit (i.e., a futile liver resection). We sought to characterize the factors associated with early recurrence after major hepatectomy. If predictive factors for futility could be identified preoperatively, this information would aid in surgical decision making and improve patient counseling to prevent futile hepatectomies.

\section{MATERIALS AND METHODS}

\section{Data Collection and Inclusion Criteria}

Our prospectively maintained database of metastatic colorectal cancer patients was queried for this study. All sequential liver resections performed for metastatic colorectal adenocarcinoma between January 1995 and May 2019 were eligible for inclusion in this analysis. All patients were staged by high-resolution contrast-enhanced cross-sectional imaging of the chest, abdomen, and pelvis, i.e. computed tomography (CT) scanning or magnetic resonance imaging with or without positron emission tomography (PET) scanning. Patients were included if they had liver-only disease (all detectable cancer confined to the liver and colon/rectum) or liver-dominant disease (minimal extrahepatic and extracolonic disease that that was rendered invisible or metabolically inactive by induction chemotherapy). Patients were excluded for widely metastatic disease or resection for palliative intent. Systemic and local therapies used in conjunction with liver resections were captured and recorded. Patients in the dataset were later monitored with serum CEA levels before and after resection. Synchronous metastases were defined as being diagnosed within 12 months of the original colorectal primary tumor diagnosis. The study was approved by the Institutional Review Board.

Variables extracted from the database included demographic and baseline health information, including date of birth, race (grouped as Caucasian, African American, Hispanic, and Asian), sex, comorbidities, social history, and body mass index. Data points related to the colorectal primary cancer were also collected, including date of diagnosis, date of resection, location of tumor, oncologic procedure, and preoperative serum CEA levels $(\mathrm{U} / \mathrm{mL})$. Pathologic data acquired included tumor size (centimeters), 
TNM classification, number of resected and positive nodes, resection margin status, and presence of perineural invasion or lymphovascular invasion. Data pertaining to the liver metastases that were extracted included the date of liver diagnosis, tumor location, date of surgery, operation performed, use of portal vein embolization, presence of extrahepatic disease, use of adjuvant therapies, CEA levels both prior to surgery and postoperatively, margin status, and the size of the tumor on preoperative CT scan (centimeters). While molecular mutation data were available for most tumors resected in the final 3 years of the data range, this was not a large enough percentage to be statistically useful. A margin was considered negative if it was wider than $1 \mathrm{~mm}$. Postoperative complications were recorded according to the Clavien-Dindo classification.

Surgical resection was only considered in the setting of extrahepatic disease in cases where a small tumor burden was present that responded to induction chemotherapy on cross-sectional imaging. In most cases, PET scanning was utilized to ensure that these disease sites were no longer hypermetabolic. A long progression-free interval was also an important factor in determining surgical eligibility in cases of extrahepatic disease. Most commonly, these sites were lung, intra-abdominal lymph nodes, or locoregional recurrence of the primary cancer within the mesocolon.

5-fluorouracil or capecitabine were administered to nearly all patients in the study per institutional protocols. For cases after 2002, oxaliplatin was added to the combination regimens of FOLFOX or XELOX, with irinotecan utilized as a second-line option within FOLFIRI regimens in instances where oxaliplatin was intolerable or ineffective. These therapies were offered to patients in the adjuvant setting for all resected stage III colorectal cancers or stage II with high-risk features as determined by multidisciplinary discussion, and again at presentation of liver metastases. In stage IV disease, these regimens were used as induction agents. Monoclonal vascular endothelial growth factor (VEGF) or epidermal growth factor receptor (EGFR) inhibitors were added for stage IV disease, beginning in 2009. After 2017, patients with excellent performance status and liver metastases were considered for treatment with FOLFOXIRI regimens. The decision to offer adjuvant systemic therapy after resection of liver metastases in previously treated patients was left to the discretion of the treating medical oncologist.

Recurrence-free survival, overall survival, diseasespecific survival, and futile liver resection were calculated from the date of operation to the date of progression or death (event), or to the date of the last follow-up visit (censored). Futile liver resection was defined as having a recurrence event within 6 months of date of operation, censoring those who died without disease over that period. Date of death was obtained from either the medical records or the Social Security Death Index. Follow-up was obtained from all patients and was updated until 1 July 2019. Standard follow-up for these patients included CT chest with triphasic CT of the abdomen by liver protocol and a CEA level every 3 months for the first year after resection and then every 6 months for years 2, 3, and 4, then yearly thereafter. Suspicious lesions seen on CT were verified as a recurrence by CT/PET scan and/or biopsy of the recurrent lesion.

\section{Statistical Analysis}

All statistical analyses were performed using SPSS version 26 (IBM Corporation, Armonk, NY, USA), and $p$ values $<0.05$ were considered significant. All tests used were two-tailed. Categorical data are expressed as frequencies and percentages, whereas continuous data are expressed as median and interquartile range (IQR). Comparison of categorical variables among groups was performed using Chi-square testing, and comparison of nonparametric variables between groups was performed using the Mann-Whitney $U$ test, as appropriate. Independent samples $t$-tests and analysis of variance testing were used to compare means of continuous variables, while multivariable logistic regression was utilized for the development of an adjusted model for futility. KaplanMeier testing was used for survival analyses for metastatic hepatectomy.

\section{RESULTS}

\section{Demographic Information}

A total of 259 major liver resections were included in the analysis. The median age was 61.3 years (IQR 53.2-68.5) and 138 patients (53\%) were male. African Americans represented $10.4 \%$ of the total sample. The median number of liver tumors was 3.0 (IQR 2.0-4.0) and the median size of the largest lesion was $4.5 \mathrm{~cm}$ (IQR 3.0-6.5). A majority (65\%) of liver metastases were synchronous (liver diagnosis within 12 months of colorectal primary). The median colorectal-to-hepatic DFI was 27.5 months for non-synchronous tumors. With respect to colorectal primary tumors, $39(15.1 \%)$ were stage $\mathrm{T} 1$ or $\mathrm{T} 2$, $193(74.8 \%)$ were stage T3, and $26(10.1 \%)$ were stage T4. Eighty-four tumors $(32.6 \%)$ were within the right colon and $55(21.3 \%)$ were rectal in origin. Metastases were confined to the right lobe only in most cases [108 (41.7\%)]. Left lobe-only or caudate-only disease represented 22 $(12.7 \%)$ and $3(1.2 \%)$ cases, respectively, while bilobar disease was seen in $81(31.3 \%)$ patients. 


\section{Systemic Therapy}

Chemotherapy was administered as part of the treatment of colorectal primaries in $77.9 \%$ of cases. Of all patients, $78.0 \%$ received chemotherapy prior to undergoing liver resection. The median $\mathrm{CEA}$ at diagnosis of metastases across the 179 patients who had the tumor marker drawn was 16.6 (IQR 7.2-67.1). Of the $150(83.8 \%)$ patients with an abnormal CEA $(>5 \mathrm{ng} / \mathrm{mL})$ at the time of liver diagnosis, a reduction in tumor marker concentration was seen in $87.9 \%$ of patients after neoadjuvant chemotherapy, and this decrease was $>75 \%$ of initial serum levels in $15.2 \%$ of patients. Of patients with abnormal CEA levels, $12.1 \%$ demonstrated increasing tumor marker levels in spite of chemotherapy. Of note, 26 patients $(10.0 \%)$ were classified as 'liver-dominant', with some degree of extrahepatic and extracolonic disease evident on staging work-up that regressed with systemic therapy.

\section{Hepatic Surgical Therapy}

Left hepatic lobectomy was performed in 50 patients (19.3\%) and right lobectomy was performed in 146 patients $(56.4 \%)$. Another 63 patients $(24.3 \%)$ underwent extended hepatectomy, while $11.2 \%$ of patients underwent preoperative portal vein embolization prior to liver resection to promote hypertrophy of the hepatic remnant. Perioperative complications were experienced in $46.2 \%$ of cases, $24.0 \%$ minor grade and $23.0 \%$ major grade (Table 1 ); $88.6 \%$ of patients had a margin-negative (R0) resection. Thirty-day mortality was $1.9 \%$.

\section{Oncologic Outcomes and Futile Liver Resection}

The analysis revealed that $12.6 \%$ (26) of patients at risk experienced a futile liver resection, with a recurrence event within 6 months of operation. The futile group was similar $(p>0.05)$ to the therapeutic hepatectomy group by univariate testing for most variables, but the futile cohort had a greater frequency of extrahepatic disease $(23.1 \%$ vs. $7.2 \%$; $p=0.009)$, portal vein embolization (28.6\% vs. $9.4 \%$; $p=0.011)$, and need for extended hepatectomy (46.2\% vs. $22.1 \% ; p=0.008$ ) (Table 2). More than three liver lesions were seen more commonly in the futile group than in the therapeutic group $(50.0 \%$ vs. $16.9 \% ; p<0.001)$. After multivariable regression, extrahepatic disease (odds ratio [OR] 5.6; $p=0.004$ ), more than three liver lesions (OR $4.9 ; p=0.001$ ), and need for extended hepatectomy (OR 2.6; $p=0.038$ ) were significant predictors of futility after liver resection. Of those patients who presented with extrahepatic disease, only $16.7 \%$ of recurrences occurred at the same site as preoperative location of disease.
After a median follow-up of 16.1 months, the median recurrence-free survival was 17.2 months (95\% confidence interval [CI] 17.0-24.2). After 60 months, $17.6 \%$ of patients at risk remained alive without disease (Table 3). Recurrence involved the liver in $60.7 \%$ of cases and the lung in $33.8 \%$ of cases. The median overall survival was 35.0 months (95\% CI 29.8-40.3). The median overall survival was 11.7 months (95\% CI 7.1-16.2) for the early recurrence cohort versus 45.6 months (95\% CI 39.1-52.1) for those who did not recur within 6 months of hepatectomy $(p<0.001)$ (Fig. 1). Notably, $70.8 \%$ of early recurrences occurred within the liver remnant, while $20.8 \%$ were pulmonary metastases. Despite changes in chemotherapy regimens over the study period, no differences were found between the rate of futile resection (11.4\% vs. $17.2 \% ; p=0.225)$ or median overall survival (36.1 months vs. 32.7 months; $p=0.726$ ) between patients diagnosed before and after 2010.

When the Clinical Risk Score was compared for the futile cohort, the median score (3.0; IQR 2.0-4.0) was greater than in the therapeutic group (2.0; IQR 1.0-3.0) but this was not significant by multivariable regression $(p=0.159)$. The individual components of the score also were not significant predictors of recurrence within the 6-month interval. Among the futile resection patients, $15.8 \%$ (vs. 9.4\%) had a preoperative CEA $>200 \mathrm{ng} / \mathrm{mL}$, $50 \%$ (vs. $31.3 \%$ ) had a lesion $>5 \mathrm{~cm}$ in diameter, $72 \%$ (vs. $57.3 \%$ ) had more than one liver lesion, $57.7 \%$ (vs. $44.8 \%$ ) had a lymph node-positive colorectal primary tumor, and $72.4 \%$ (vs. 63.9\%) had a colorectal-to-hepatic DFI of $<12$ months $(p>0.05)$.

\section{DISCUSSION}

Discretion should be exercised when considering major liver resection for metastatic colorectal cancer and the high-risk features outlined in these analyses. The presence of more than three liver metastases and/or extrahepatic disease, and the need for an extended hepatectomy, suggest a substantial likelihood of a nontherapeutic resection, and less morbid alternative therapies should be considered in patient discussions. Any attempt at a major liver resection, even with preoperative chemotherapy, may well fail to achieve durable disease control and may preclude further liver-directed therapies with significant parenchymal and functional loss. A large operation may also delay additional systemic therapy and worsen outcomes, especially in the event of a complication. These preoperative clinical features are crucial considerations for surgical decision making. 
TABLE 1 Characteristics of futile liver resection patients with multivariable adjusted odds ratios

\begin{tabular}{|c|c|c|c|c|c|c|}
\hline Variable & $\begin{array}{l}\text { Therapeutic } \\
\text { resection }[n(\%)]\end{array}$ & $\begin{array}{l}\text { Futile } \\
\text { resection }[n(\%)]\end{array}$ & $p$-Value & $\begin{array}{l}\text { Adjusted } \\
\text { OR }\end{array}$ & $95 \%$ CI & $p$-Value \\
\hline Female & $81(44.8)$ & $14(53.8)$ & 0.384 & & & \\
\hline African American & $17(10.1)$ & $3(12.0)$ & 0.728 & & & \\
\hline Right-sided colon lesion & $58(32.0)$ & $11(42.3)$ & 0.299 & & & \\
\hline Synchronous colon and liver & $115(63.9)$ & $21(72.4)$ & 0.372 & & & \\
\hline Chemotherapy for primary & $128(80.0)$ & $15(71.4)$ & 0.365 & & & \\
\hline Extrahepatic disease & $13(7.2)$ & $6(23.1)$ & 0.009 & 5.6 & $1.8-18.1$ & 0.004 \\
\hline Left lobe lesion & $31(17.1)$ & $5(19.2)$ & 0.791 & & & \\
\hline Right lobe lesion & $132(72.9)$ & $19(73.1)$ & 0.987 & & & \\
\hline Bilobar disease & $19(10.5)$ & $5(19.2)$ & 0.193 & & & \\
\hline More than three liver lesions & $30(16.8)$ & $13(50.0)$ & $<0.001$ & 4.9 & $2.0-12.4$ & 0.001 \\
\hline Maximum liver CEA $>5.0$ & $105(82.0)$ & $18(94.7)$ & 0.162 & & & \\
\hline$>50 \%$ reduction in CEA with chemotherapy & $18(37.5)$ & $2(25)$ & 0.495 & & & \\
\hline Portal vein embolization & $14(9.4)$ & $6(28.6)$ & 0.011 & & & \\
\hline Extended hepatectomy & $40(22.1)$ & $12(46.2)$ & 0.008 & 2.6 & $1.2-6.6$ & 0.038 \\
\hline Right lobectomy & $106(58.6)$ & $10(38.5)$ & 0.530 & & & \\
\hline Left lobectomy & $35(19.3)$ & $4(15.4)$ & 0.630 & & & \\
\hline Blood transfusion required & $54(29.8)$ & $10(38.5)$ & 0.373 & & & \\
\hline Stage $\mathrm{T} 1$ primary & $6(3.3)$ & $1(3.8)$ & 0.690 & & & \\
\hline Stage $\mathrm{T} 2$ primary & $24(13.3)$ & $3(11.5)$ & & & & \\
\hline Stage T3 primary & $136(75.1)$ & $19(73.1)$ & & & & \\
\hline Stage $\mathrm{T} 4$ primary & $15(8.3)$ & $3(11.5)$ & & & & \\
\hline Lymph node positive primary & $83(57.6)$ & $14(63.6)$ & 0.595 & & & \\
\hline \multirow[t]{2}{*}{ History of liver disease } & $7(3.9)$ & $3(11.5)$ & 0.116 & & & \\
\hline & Mean (SD) & Mean (SD) & & & & \\
\hline Age, years & $60.7(11.0)$ & $57.5(11.4)$ & 0.187 & & & \\
\hline Body mass index, $\mathrm{kg} / \mathrm{m}^{2}$ & $27.3(5.6)$ & $26.1(5.6)$ & 0.307 & & & \\
\hline CEA primary diagnosis, $\mathrm{ng} / \mathrm{mL}$ & $89.3(288.6)$ & $197.8(479.8)$ & 0.191 & & & \\
\hline CEA hepatic diagnosis, $\mathrm{ng} / \mathrm{mL}$ & $102.6(291.4)$ & $179.7(450.0)$ & 0.322 & & & \\
\hline Size of largest liver lesion, $\mathrm{cm}$ & $5.0(3.2)$ & $5.6(2.7)$ & 0.387 & & & \\
\hline
\end{tabular}

OR odds ratio, $C I$ confidence interval, $C E A$ carcinoembryonic antigen, $S D$ standard deviation

TABLE 2 Perioperative outcomes after major hepatectomy

\begin{tabular}{llllc}
\hline Variable & $\begin{array}{l}\text { Therapeutic } \\
\text { resection }[n(\%)]\end{array}$ & $\begin{array}{l}\text { Futile } \\
\text { resection }[n(\%)]\end{array}$ & $p$-Value & Overall \% \\
\hline Positive margin & $20(11.4)$ & $3(11.5)$ & 0.987 & 11.4 \\
Major complication (grade 3-5) & $40(22.1)$ & $6(23.1)$ & 0.911 & 23.0 \\
Minor complication (grade 1, 2) & $31(24.3)$ & $3(21.9)$ & 0.911 & 24.0 \\
Overall morbidity & $71(46.4)$ & $9(45.0)$ & 0.906 & 46.2 \\
30-day mortality & & & & 1.9 \\
\hline
\end{tabular}

Of this large experience of major liver resections, $12.6 \%$ were ultimately futile. It is a humbling realization that, in spite of the best judgment of multiple high-volume surgical oncologists, more than 1 in every 10 major liver resections provided no effective disease control and subjected patients to undue risk. Interestingly, despite advances in chemotherapeutic regimens and increasing options for liver-directed therapies over the study period, the rate of futile resection and overall survival after liver resection did not appear to change. This highlights that surgical selection is still of paramount importance in the complex management of liver-only or liver-dominant stage IV colorectal 
TABLE 3 Long-term outcomes after hepatectomy

\begin{tabular}{lllr}
\hline Variable & $\begin{array}{l}\text { Therapeutic } \\
\text { resection }[n(\%)]\end{array}$ & $\begin{array}{l}\text { Futile } \\
\text { resection }[n(\%)]\end{array}$ & $p$-Value \\
\hline Dead of disease & $86(47.5)$ & $25(96.2)$ & $<0.001$ \\
Alive with disease & $23(12.7)$ & $1(3.8)$ & $<0.001$ \\
No evidence of disease & $50(27.6)$ & $0(0.0)$ & 0.001 \\
Dead of other cause & $22(12.2)$ & $0(0.0)$ & 0.084 \\
Liver recurrence & $65(58.0)$ & $17(70.8)$ & 0.245 \\
Lung recurrence & $42(37.5)$ & $5(20.8)$ & 0.119 \\
Lymph node recurrence & $17(15.2)$ & $2(8.3)$ & 0.526 \\
Peritoneal recurrence & $14(12.5)$ & $2(8.3)$ & 0.565 \\
\hline
\end{tabular}

FIG. 1 Survival functions by Kaplan-Meier estimation demonstrating overall survival (in months) of the futile cohort (red) [11.7 months; 95\% CI 7.1-16.2] versus the therapeutic resection cohort (top panel, blue) [45.6 months; 95\% CI $39.1-52.1]$ and the non-futile recurrence patients (bottom panel, blue) [39.6 months; 95\% CI 32.9-46.3; $p<0.001]$. CI confidence interval
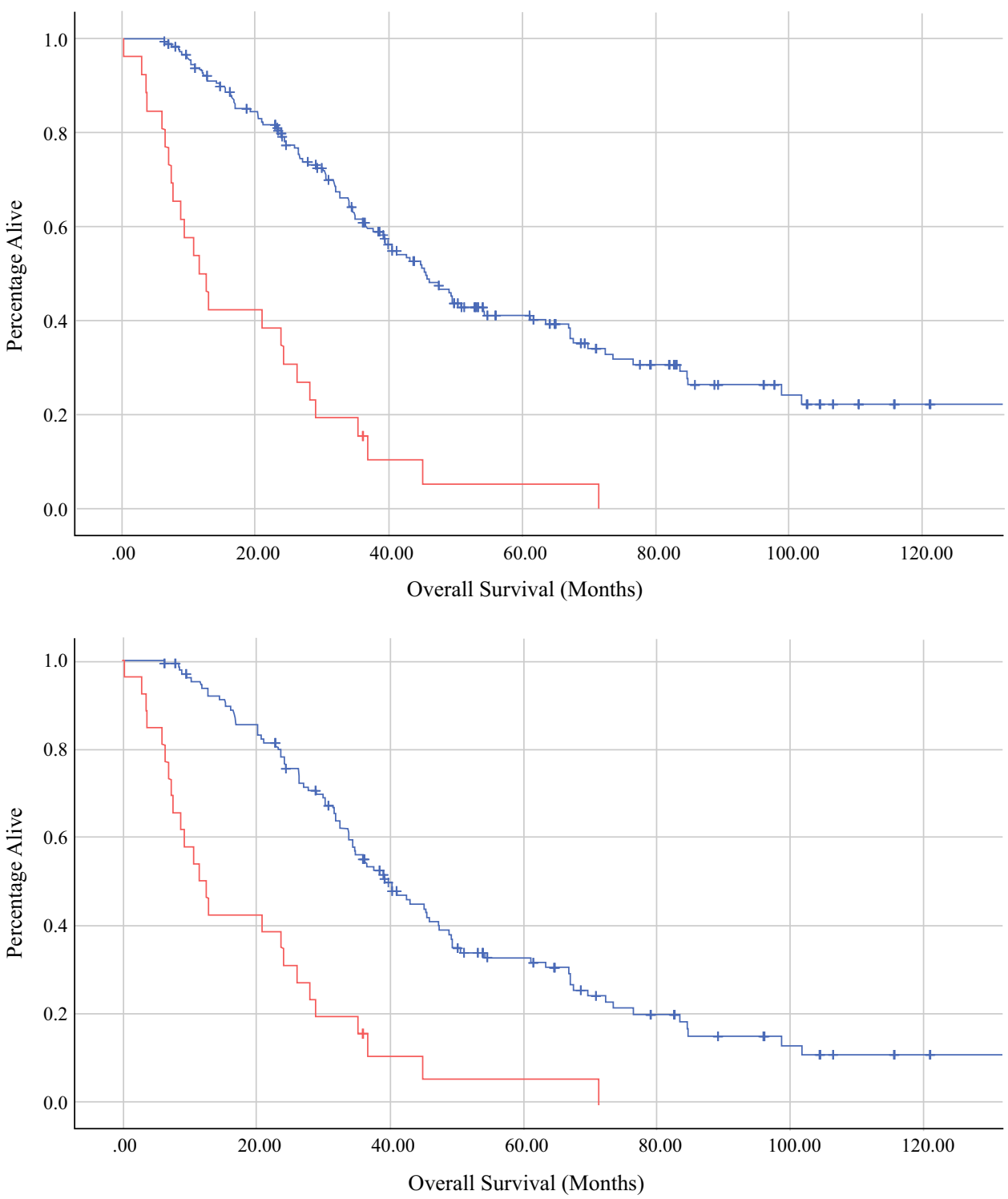

cancer. Indeed, our prospectively maintained database allowed for an analysis of numerous specific clinical, histologic, and oncologic variables.
The study's definition for a futile liver resection was a recurrence event within 6 months of operation. We felt that this was a cleaner definition of futile for the modern era, considering that many alternative and adjuvant therapies 
affect survival and can obfuscate the impact of the surgery. In our opinion, a rapid recurrence after a major liver resection represents a failure of patient selection. Six months is an exceedingly short post-hepatectomy DFI and it is difficult to claim any oncologic benefit from the operation. Several previous studies have suggested that post-hepatectomy recurrence before 6-8 months has negative implications for overall survival. ${ }^{25,29}$ Our data were consistent with this finding - a median survival of only 11.7 months was seen in the futile group versus 45.6 months for therapeutic resections. The drastically reduced life expectancy suggests that many of these progression events are not easily salvageable with further therapies. Moreover, it is probable that early recurrences are a consequence of less favorable disease biology that also negatively impacts prognosis.

Patients who present with more than three liver lesions are nearly fivefold as likely to experience a futile liver resection, which implies that numerous clinically apparent liver lesions are likely accompanied by micrometastases outside of the resection field that may blossom postoperatively. Furthermore, this disease presentation may signify a disease biology that will see improved outcomes without long interruptions in systemic therapy, as would be expected around a major abdominal surgery. This result is similar to previous studies that concluded the presence of between four and eight lesions is associated with earlier recurrences. $^{25,26,28}$

The need for extended hepatectomy and the presence of extrahepatic disease are important indicators of the distribution of disease burden. An extended hepatectomy in order to remove all apparent disease in the liver suggests significant bilobar involvement. This also predicts a more diminutive hepatic remnant that may tolerate fewer future liver-directed therapies should a recurrence occur. Extrahepatic disease, in most cases, is a clinical diagnosis made with imaging that showed a response to chemotherapy. As only $17 \%$ of patients with this finding recurred at the same site that was diagnosed preoperatively, the presence of this factor serves more as marker of widely disseminated smallvolume disease that is not durably controlled with systemic therapy. Signs of a more widespread disease distribution are important in the surgical assessment of major liver resection candidates.

While the Fong Clinical Risk Score is a validated means of predicting disease recurrence and survival after hepatic metastasectomy for colorectal cancer, the factors in the score are not designed for surgical estimation of futility risk. Many liver resections that ultimately result in a recurrence provide at least a transitory disease control benefit, and many delayed recurrences are salvageable with further therapies. In our analysis, none of the individual components of the score were shown to be significant predictors of futility. Another prior study concluded that a node-positive primary and metastasis diameter $>3 \mathrm{~cm}$ may be of some benefit in predicting short-interval recurrence, but this was not seen in our multivariable comparison. ${ }^{27}$ The shortcomings in predicting futility of models designed to predict any recurrence event underscore the need for dedicated short-interval predictors for treatment planning.

This study has several limitations. Naturally, the singlecenter nature of this investigation may make the findings less generalizable. In most cases, our institutional bias is to provide chemotherapy prior to a major liver resection, as $78 \%$ of patients in the dataset received pre-hepatectomy chemotherapy. The study is also retrospective and will always suffer from some degree of selection bias. Multivariable models were utilized to correct for this but the effects can never be eliminated completely. As a database study, problems with data entry and maintenance may occur even under the best circumstances. In addition, the extended time period included in the study may combine management strategies from different eras and evolving systemic therapy regimens. In spite of these faults, the consistent reporting of clinical variables and the large patient sample reinforce confidence in the study conclusions.

\section{CONCLUSION}

A deliberate approach with risk stratification for futility is recommended to avoid potentially harmful liver resections in metastatic colorectal cancer and provide accurate expectations to patients. A rapid recurrence soon after the operation is a clear failure of surgical therapy to achieve the intended disease control. With $70.8 \%$ of early recurrences occurring within the liver, aggressive parenchymal resection may burn bridges for other potential therapies. $^{27,29}$ Short-interval recurrence has a dismal prognosis that may be due to more aggressive disease biology, chemotherapy resistance, or small liver remnants not eligible for further liver-directed therapies. Fortunately, clinical characteristics exist that allow surgeons to predict a futile major hepatectomy in the preoperative setting. Attention to such high-risk features is important for multidisciplinary discussions to optimize treatment sequence and selection in advanced colorectal cancer and can aid in difficult discussions with patients and families.

\section{ACKNOWLEDGMENTS None.}

AUTHOR CONTRIBUTIONS Study conception and design: MWF, CRS, MEE, PP, RCGM, Acquisition of data: MWF, RCGM, Analysis and interpretation of data: MWF, CRS, MEE, PP, KMM, RCGM, Drafting of the manuscript: MWF, CRS, MEE, PP, KMM, RCGM, Critical revision: MWF, CRS, MEE, PP, KMM, RCGM

DISCLOSURE None declared. 


\section{REFERENCES}

1. Siegel RL, Miller KD, Jemal A. Cancer statistics, 2019. $C A$ Cancer J Clin. 2019;69(1):7-34.

2. Hackl C, Neumann P, Gerken M, Loss M, Klinkhammer-Schalke M, Schlitt HJ. Treatment of colorectal liver metastases in Germany: a ten-year population-based analysis of 5772 cases of primary colorectal adenocarcinoma. BMC Cancer. 2014;14:810.

3. Manfredi S, Lepage C, Hatem C, Coatmeur O, Faivre J, Bouvier AM. Epidemiology and management of liver metastases from colorectal cancer. Ann Surg. 2006;244(2):254-9.

4. Cummings LC, Payes JD, Cooper GS. Survival after hepatic resection in metastatic colorectal cancer: a population-based study. Cancer. 2007;109(4):718-26.

5. Pulitano C, Castillo F, Aldrighetti L, Bodingbauer M, Parks RW, Ferla G, et al. What defines "cure" after liver resection for colorectal metastases? Results after 10 years of follow-up. $H P B$ (Oxford). 2010;12(4):244-9.

6. Adam R, Yi B, Innominato PF, Barroso E, Laurent C, Giuliante $\mathrm{F}$, et al. Resection of colorectal liver metastases after second-line chemotherapy: is it worthwhile? A LiverMetSurvey analysis of 6415 patients. Eur J Cancer. 2017;78:7-15.

7. Fong Y, Fortner J, Sun RL, Brennan MF, Blumgart LH.(1999) Clinical score for predicting recurrence after hepatic resection for metastatic colorectal cancer: analysis of 1001 consecutive cases. Ann Surg. 230(3):309-318; discussion 318-321.

8. Hill CR, Chagpar RB, Callender GG, Brown RE, Gilbert JE, Martin RC 2nd, et al. recurrence following hepatectomy for metastatic colorectal cancer: development of a model that predicts patterns of recurrence and survival. Ann Surg Oncol. 2012;19(1):139-44.

9. Chua TC, Saxena A, Chu F, Zhao J, Morris DL. Predictors of cure after hepatic resection of colorectal liver metastases: an analysis of actual 5- and 10-year survivors. J Surg Oncol. 2011;103(8):796-800.

10. Tan MC, Butte JM, Gonen M, Kemeny N, Fong Y, Allen PJ, et al. Prognostic significance of early recurrence: a conditional survival analysis in patients with resected colorectal liver metastasis. HPB (Oxford). 2013;15(10):803-13.

11. Bhogal RH, Hodson J, Bramhall SR, Isaac J, Marudanayagam R, Mirza DF, et al. Predictors of early recurrence after resection of colorectal liver metastases. World J Surg Oncol. 2015;13:135.

12. Kaibori M, Iwamoto $\mathrm{Y}$, Ishizaki M, Matsui K, Yoshioka K, Asano $\mathrm{H}$, et al. Predictors and outcome of early recurrence after resection of hepatic metastases from colorectal cancer. Langenbecks Arch Surg. 2012;397(3):373-81.

13. Giacchetti S, Perpoint B, Zidani R, Le Bail N, Faggiuolo R, Focan C, et al. Phase III multicenter randomized trial of oxaliplatin added to chronomodulated fluorouracil-leucovorin as firstline treatment of metastatic colorectal cancer. J Clin Oncol. 2000;18(1):136-47.

14. Hurwitz H, Fehrenbacher L, Novotny W, Cartwright T, Hainsworth J, Heim W, et al. Bevacizumab plus irinotecan, fluorouracil, and leucovorin for metastatic colorectal cancer. $N$ Engl J Med. 2004;350(23):2335-42.

15. Neugut AI, Lin A, Raab GT, Hillyer GC, Keller D, O'Neil DS, et al. FOLFOX and FOLFIRI Use in Stage IV colon cancer: analysis of SEER-medicare data. Clin Colorectal Cancer. 2019;18(2):133-40.

16. Vargas GM, Sheffield KM, Parmar AD, Han Y, Gajjar A, Brown $\mathrm{KM}$, et al. Trends in treatment and survival in older patients presenting with stage IV colorectal cancer. J Gastrointest Surg. 2014;18(2):369-77.

17. Tanaka T, Sato T, Nishiofuku H, Masada T, Tatsumoto S, Marugami N, et al. Selective TACE with irinotecan-loaded 40 mum microspheres and FOLFIRI for colorectal liver metastases: phase I dose escalation pharmacokinetic study. BMC Cancer. 2019;19(1):758.

18. Wang LJ, Zhang ZY, Yan XL, Yang W, Yan K, Xing BC. Radiofrequency ablation versus resection for technically resectable colorectal liver metastasis: a propensity score analysis. World J Surg Oncol. 2018;16(1):207.

19. Martin RC 2nd, Scoggins CR, Schreeder M, Rilling WS, Laing CJ, Tatum CM, et al. Randomized controlled trial of irinotecan drug-eluting beads with simultaneous FOLFOX and bevacizumab for patients with unresectable colorectal liver-limited metastasis. Cancer. 2015;121(20):3649-58.

20. Matsuoka H, Morise Z, Tanaka C, Hayashi T, Ikeda Y, Maeda K, et al. Repeat hepatectomy with systemic chemotherapy might improve survival of recurrent liver metastasis from colorectal cancer-a retrospective observational study. World J Surg Oncol. 2019;17(1):33.

21. Wang SJ, Si XY, Cai ZB, Zhou YM. Survival after repeat hepatectomy for recurrent colorectal liver metastasis: a review and meta-analysis of prognostic factors. Hepatobiliary Pancreat Dis Int. 2019;18(4):313-20.

22. Wicherts DA, de Haas RJ, Salloum C, Andreani P, Pascal G, Sotirov D, et al. Repeat hepatectomy for recurrent colorectal metastases. Br J Surg. 2013;100(6):808-18.

23. Fromer MW, Aloia TA, Gaughan JP, Atabek UM, Spitz FR. The utility of the MELD score in predicting mortality following liver resection for metastasis. Eur J Surg Oncol. 2016;42(10):1568-75.

24. Kasai M, Cipriani F, Gayet B, Aldrighetti L, Ratti F, Sarmiento JM, et al. Laparoscopic versus open major hepatectomy: a systematic review and meta-analysis of individual patient data. Surgery. 2018;163(5):985-95.

25. Imai K, Allard MA, Benitez CC, Vibert E, Sa Cunha A, Cherqui $D$, et al. Early recurrence after hepatectomy for colorectal liver metastases: what optimal definition and what predictive factors? Oncologist. 2016;21(7):887-94.

26. Malik HZ, Gomez D, Wong V, Al-Mukthar A, Toogood GJ, Lodge JP, et al. Predictors of early disease recurrence following hepatic resection for colorectal cancer metastasis. Eur J Surg Oncol. 2007;33(8):1003-9.

27. Lin J, Peng J, Zhao Y, Luo B, Zhao Y, Deng Y, et al. Early recurrence in patients undergoing curative resection of colorectal liver oligometastases: identification of its clinical characteristics, risk factors, and prognosis. $J$ Cancer Res Clin Oncol. 2018;144(2):359-69.

28. Takahashi S, Konishi M, Kinoshita T, Gotohda N, Kato Y, Saito $\mathrm{N}$, et al. Predictors for early recurrence after hepatectomy for initially unresectable colorectal liver metastasis. J Gastrointest Surg. 2013;17(5):939-48.

29. Takahashi S, Konishi M, Nakagohri T, Gotohda N, Saito N, Kinoshita T. Short time to recurrence after hepatic resection correlates with poor prognosis in colorectal hepatic metastasis. Jpn J Clin Oncol. 2006;36(6):368-75.

Publisher's Note Springer Nature remains neutral with regard to jurisdictional claims in published maps and institutional affiliations. 